\title{
Über die photoelektrische Emission positiver Ionen aus einer Adsorptionsschidht
}

\author{
Von H. Moesta \\ Aus dem Institut für Physikalische Chemie der Universität Bonn \\ (Z. Naturforschg. 17 a, 578-584 [1962] ; eingegangen am 14. April 1962)
}

\begin{abstract}
Es wird über einen photoelektrischen Effekt mit positiven Ionen berichtet, der an einer Schicht von auf Platin adsorbiertem Kalium beobachtet werden kann.

Bei Belichtung einer solchen Schicht wird der von der Oberfläche des Platins fließende thermische Ionenstrom geändert. In gewissen Bereichen der Energieverteilung der emittierten Ionen tritt eine Photoemission zusätzlich zur thermischen Emission auf. In anderen Bereichen der Energieverteilung ist eine durch das Licht verursachte Verkleinerung des thermischen Ionenstromes zu beobachten. Beide Effekte treten auch noch bei Wellenlängen auf, die größer sind als zur Ionisation von Kalium in der Gasphase erforderlich ist. Die Apparatur wird beschrieben und Ergebnisse diskutiert.
\end{abstract}

An einer polykristallinen Platin-Oberfläche adsorbiertes Kalium liegt dort als Gemisch von Ionen und mehr oder weniger stark polarisierten Atomen vor ${ }^{1-3}$.

Beide Komponenten erzeugen Oberflächendipole ${ }^{4}$. Bei genügend hoher Temperatur und einem in geeigneter Richtung angelegten elektrischen Feld fließt von einer solchen Oberfläche ein thermischer Ionenstrom.

Man kann sich leicht überlegen, daß für den Ablauf der Oberflächenreaktion

$$
\mathrm{K}_{\text {adsorbiert }} \rightarrow \mathrm{K}_{\text {desorbiert }}^{+}+\text {Elektron }_{\text {metall }},
$$

die der Bildung und Desorption eines Ions an der Metalloberfläche entspricht, der Übergang eines Elektrons zwischen Metall und Adsorbat maßgebend ist. Es konnte in einer früheren Arbeit gezeigt werden, daß dieser Übergang selbst dann nicht ohne Hemmung verläuft, wenn ein erheblicher Energiegewinn mit dem Ablauf der Reaktion nach rechts verbunden ist $^{2}$.

Um die Natur dieser Hemmung näher zu untersuchen, wurde versucht, durch Einstrahlung von Licht die Verhältnisse in der Adsorptionsschicht zu ändern.

Die Einstrahlung von Licht kann auf folgende Weisen die genannten Verhältnisse beeinflussen:

a) Die Oberflächendipole können Schwingungsenergie aus der Strahlung aufnehmen.

1 Sh. Datz u. E. H. Taylor, J. Chem. Phys. 25, 389 [1956].

2 H. Moesta, Z. Naturforschg. 16 a, 288 [1961]; Z. Elektrochem. 61, 1235 [1957].

3 Die Verhältnisse an Wolfram wurden in neuerer Zeit von F. L. Hughes, Phys. Rev. 113, 1036 [1959] und F. L. Hughes und H. Levinstein, Phys. Rev. 113, 1029 [1959] genauer untersucht. b) Die Besetzung der elektronischen Oberflächenzustände des Metalles kann sich ändern.

c) Das adsorbierte Kalium-Atom oder ein aus dem Kalium-Atomrumpf und einem in der Nähe lokalisierten Elektron bestehender "Adsorptionskomplex “ * kann zunächst elektronisch angeregt werden und dann sein Elektron aus dem angeregten Zustand mit einer anderen Wahrscheinlichkeit abgeben.

In jedem dieser Fälle wird sich der auf der oben angeführten Reaktion beruhende Ionenstrom ändern. Analog zum lichtelektrischen Effekt, bei dem Elektronen aus dem Metall ausgelöst werden, sollte es hier möglich sein, eine Photo-Emission positiver Ionen zu beobachten.

\section{Apparatur}

Zum Nachweis der Existenz eines solchen Effektes kann man keine einfache Dioden-Anordnung, wie beispielsweise bei den herkömmlichen Photozellen, benutzen. Bei den erforderlichen hohen Lichtintensitäten und in Gegenwart von Kaliumdampf werden an allen Elektroden Photoelektronen freigemacht, die die Messungen verfälschen würden. Auch ein Faraday-Käfig als Auffänger läßt sich nicht benutzen, sofern er nicht vollständig vor Licht geschützt wird.

Aus diesen Gründen erschien zur selektiven Erfassung der emittierten Ionen die Anwendung eines Magnetfeldes erforderlich. Ein Magnetfeld gestattet außer der Trennung von Elektronen und Ionen eine Geschwin-

${ }^{4}$ Siehe z. B. R. W. Gurney, Phys. Rev. 47, 479 [1935] sowie J. A. Becker, Advances in Catalysis VII, Academic Press, New York 1955.

* I. N. Dobrezow, Elektronen und Ionenemission, VEB Technik, Berlin 1954, S. $202 \mathrm{ff}$. 
digkeitsfokussierung, die im Hinblick auf zu erwartende Unterschiede in der Geschwindigkeitsverteilung zwischen thermisch und optisch emittierten Ionen von Vorteil erschien.

Es wurde ein $180^{\circ}$-Elektromagnet mit einem Polschuh-Durchmesser von $130 \mathrm{~mm}$ und einem Luftspalt von $50 \mathrm{~mm}$ gewählt, um die Lichteinstrahlung mit genügend großem Öffnungswinkel vornehmen zu können.

Abb. 1 zeigt eine schematische Darstellung der verwendeten Apparatur.

Als Photoanode (Ionenquelle) diente die innere Oberfläche einer Hohlkugel von $40 \mathrm{~mm}$ Durchmesser aus technisch reinem Platin. Einstrahlung des Lichtes und Extraktion der Ionen erfolgte durch eine Öffnung von $20 \mathrm{~mm}$ Durchmesser, während durch eine kleinere, um $90^{\circ}$ versetzte Bohrung Kalium-Dampf aus einem Ofen eingelassen werden konnte. In die große Öffnung ragt eine zylindrische Ziehblende (Z) von $16 \mathrm{~mm}$ lichter Weite $3 \mathrm{~mm}$ tief hinein. Gegenüber der großen Öffnung befindet sich zur Justierung noch eine Bohrung von 0,5 mm Durchmesser.

Die Platinkugel kann mittels einer Wicklung (W) geheizt werden, die Temperaturmessung der Kugel erfolgt mit einem Thermoelement. Diese Anordnung wurde gewählt, um eine mehrfache Reflexion des in die Kugel eingestrahlten Lichtes zu erzielen.

Ein von der Innenwand der Kugel ausgehender Ionenstrom wird durch die Ziehblende extrahiert und tritt in das Trennfeld des Magneten ein.

Neben der Platinkugel erkennt man in der Abb. den Ofen (Of) für den Kalium-Dampfstrahl. In diesem Ofen befindet sich das Kalium in einer geschlossenen Pyrex-Ampulle, die nach dem Ausheizen der Apparatur mit dem Stößel (St) zerschlagen wird. Dieser Stößel trägt gleichzeitig eine Blende, mit der die Öffnung des Ofens verschlossen werden kann, um den Dampfstrahl zu unterbrechen. Das Kalium wurde vor dem Einfüllen in die Ampulle mehrfach destilliert. Bei der Konstruktion des beschriebenen Instrumentes wurde bewußt auf hohe Auflösung verzichtet, um auf alle Fälle eine für den Nachweis des Effektes genügende Intensität zu erzielen.

Für den Nachweis des Ionenstromes dient ein 17-stufiger Kupfer-Beryllium-Multiplier (M).

Um Streulicht von der ersten Dynode des Multipliers fernzuhalten, wurden zwei Blenden (B) so angebracht, daß diese Dynode nur die Öffnung eines Hohlraumes (S) „sehen“ kann.

Die ganze Anordnung befindet sich in einem Gehäuse aus V2A-Stahl und konnte auf $200^{\circ} \mathrm{C}$ ausgeheizt werden. Das Gehäuse des Apparates besitzt in Richtung der Achse der Ziehblende zwei Quarzfenster. Vor dem größeren Fenster $\left(\mathrm{F}_{1}\right)$ befindet sich eine $\mathrm{Hg}$-HochdruckDampflampe (O s r a m HBO $200 \mathrm{Watt}$ ) und eine von einem Synchron-Motor angetriebene rotierende Blende.
Das kleinere Fenster $\left(\mathrm{F}_{2}\right)$ diente beim Zusammenbau zum Justieren der Kugel im Strahlengang. Zur Einengung des von der Lampe ausgesandten Wellenlängenbereiches standen folgende $\mathrm{S} \mathrm{ch}$ o t t - Interferenzfilter

Typ UV-IL: $316,336 \mathrm{~nm}$,

Typ AL: $\quad 403,432,495,543,588,616,775 \mathrm{~nm}$

sowie ein Glasfilter und eine Wasserküvette mit Quarzfenstern zur Verfügung. Die Durchlässigkeit der beiden letztgenannten Filter war mit einem Spektral-Photometer ausgemessen worden.

Evakuiert wurde mit einer $100 \mathrm{l} / \mathrm{s}$ Quecksilber-Diffusionspumpe. Der Druck in der Apparatur lag während der Messungen zwischen $5 \cdot 10^{-8}$ und $1 \cdot 10^{-6} \mathrm{~mm} \mathrm{Hg}$.

Die zu erwartende Größe des Photoeffektes ist gering, da die erreichbaren Bedeckungen der Platinoberfläche mit Kalium in der Größenordnung von $10^{-4}$ bis $10^{-2}$ der Monoschicht liegen. Da sich andererseits dem Photostrom ein großer Strom thermisch emittierter Ionen überlagern kann, waren Maßnahmen zur Trennung des Photostromes vom thermischen Strom, zur Unterdrückung des thermischen Rauschens der Emission sowie zur Unterdrückung des durch den großen thermischen Ionenstrom ebenfalls großen multiplikativen Rauschens des Multipliers erforderlich. Abb. 2 zeigt die hierzu benutzte Anordnung in Blockschaltung ${ }^{5}$.

Zur Trennung der Photoionenemission von der thermischen Ionen-Emission wird das Licht von der rotierenden Blende mit einer Frequenz von $17 \mathrm{~Hz}$ zerhackt. Die Wechselstromkomponente vom Ausgang des Multipliers wird zunächst in einem Breitband-Verstärker (Tektronix 53/54 D) verstärkt und über einen Hochpaß $(5 \times 1 \mu \mathrm{F}, 5 \times 1 \mathrm{M} \Omega)$ einem gesteuerten Gleichrichter zugeführt. Der Gleichrichter wird über

5 Wesentliche Ratschläge für den Bau des elektronischen Teiles verdanke ich Herrn Dipl.-Ing. Peter Herrwerth. 
eine Photozelle von der rotierenden Blende gesteuert. Die Ausgangsspannung des Gleichrichters geht über einen Tiefpaß mit einer Bandbreite von etwa $1 / 10 \mathrm{~Hz}$ auf die $y$-Achse eines $x-y$-Schreibers.

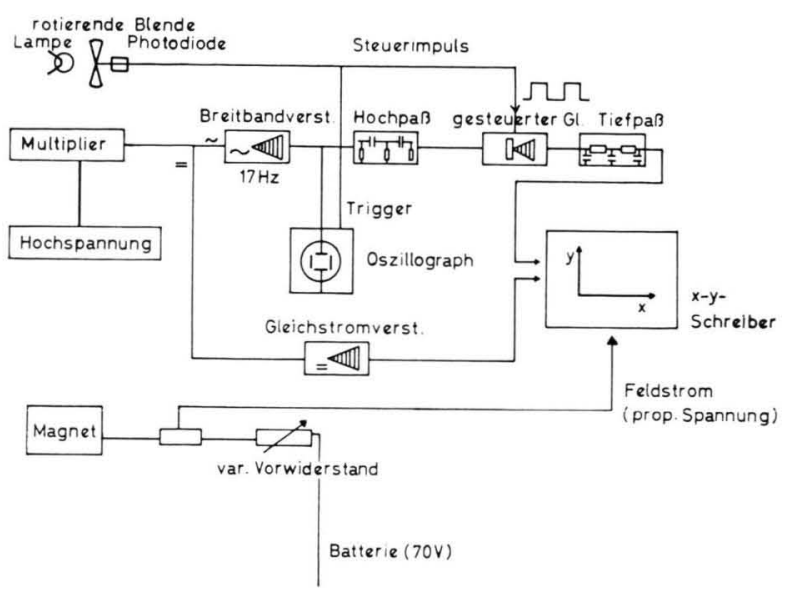

Abb. 2. Blockschaltung der Meßanordnung.

Die $x$-Achse des Schreibers wird von dem über einen motorgetriebenen Widerstand stetig variablen Feldstrom des Magneten gesteuert. Man erhält dann auf dem Schreiber unmittelbar die Abhängigkeit des Ionenstromes vom Feldstrom, d. h. vom Magnetfeld. Das Magnetfeld wurde in Abhängigkeit vom Feldstrom vermittels einer rotierenden Spule geeicht, die ihrerseits mit Protonenresonanz geeicht war ${ }^{6}$.

Das Schirmbild des Oszillographen wurde während der Messungen zur Aussteuerungs-Kontrolle des Gleichrichters benützt.

Mit dieser Anordnung kam bei $10^{-12}$ Ampere am Ausgang des Multipliers der Schreiberausschlag in die Größe des Untergrundes. Bei einer Verstärkung von $10^{+5} \mathrm{im}$ Multiplier lag die Nachweisgrenze der Anordnung also bei einem Photostrom von $10^{-17} \mathrm{~A}$.

Die Breite der so registrierten „Peaks" wird einerseits durch die Geometrie der Anordnung und die Homogenität des Magnetfeldes bestimmt, andererseits durch die Geschwindigkeitsverteilung, mit der die Ionen die Oberfläche der Platinkugel verlassen.

\section{Versuche}

Zu Beginn der Versuche wird die Apparatur ausgeheizt und auf $2-4 \cdot 10^{-8} \mathrm{~mm} \mathrm{Hg}$ evakuiert. Die Heizwicklungen von $\mathrm{Pt}$-Kugel und Kalium-Ofen werden hierbei gesondert ausgeheizt.

Am Anfang emittiert die Kugel K- und Na-Ionen, die von Verunreinigungen herstammen. Wenn diese Emission in die Größenordnung des Rauschens gesunken ist, was nach etwa $4 \mathrm{Stdn}$. bei $600{ }^{\circ} \mathrm{C}$ eintritt ${ }^{7}$,

${ }^{6}$ Die geeichte Spule wurde von Herrn Dr. Althoff, Physikalisches Institut der Universität Bonn, freundlicherweise zur Verfügung gestellt. wird die Ampulle im Ofen zerschlagen und die Apparatur ist meßbereit. Durch Einstellen der Ofentemperatur auf $120^{\circ} \mathrm{C}$ wird ein K-Dampfstrahl in die Kugel eingeblasen.

Beim Aufheizen der Platinkugel beginnt ab $380{ }^{\circ} \mathrm{C}$ ein thermischer Ionenstrom zu fließen. Dieser Ionenstrom dient zur Einstellung der Apparatur. Zunächst wird mit $20 \mathrm{~V}$ zwischen Kugel und Ziehblende das Maximum des K-Peaks eingestellt und der zugehörige Feldstrom ermittelt. Bei dieser Spannung wird auf Verunreinigungen geprüft. Es wurden lediglich $\mathrm{Na}$ Ionen beobachtet (max. $1 / 2 \%$ des K-Peaks).

Nach diesen Vorbereitungen wird nun die Ziehspannung auf beispielsweise $4 \mathrm{~V}$ erniedrigt und das Feld entsprechend geschwächt. Der K-Peak ist jetzt erheblich breiter, da die Ionen die Oberfläche mit verschiedener Geschwindigkeit verlassen. Die Größe dieser Anfangsgeschwindigkeit wurde für das Maximum des thermischen Stromes bei $500{ }^{\circ} \mathrm{C}$ Platintemperatur zu $1,7 \pm 0,2$ $\mathrm{eV}$ ermittelt, sie hängt empfindlich vom Bedeckungs$\operatorname{grad} a b$.

Die folgenden Versuche wurden alle so durchgeführt, daß nach Einstellen der Kugel- und der Ofentemperatur das Magnetfeld periodisch das gesamte Gebiet, in dem ein Ionenstrom zu beobachten war, überstrich.

Registriert wurde zunächst der Ausgang des Gleichrichters, also die $17 \mathrm{~Hz}$-Komponente des Multiplierstromes, bei Belichtung der Kugel, danach erfolgte jeweils ein Durchlauf ohne Belichtung zur Kontrolle. Nach einigen solchen Durchläufen wurde der Multiplier vom Wechselstrom-Kanal getrennt und die Gleichstromkomponente seines Ausganges mit einem Zerhackerverstärker über die bereits aufgenommenen Kurven geschrieben.

Man kann daher die Lage der Maxima bzw. Minima des vom Licht hervorgerufenen Stromes relativ zum thermischen Strom unmittelbar den Abbildungen entnehmen. Die Vorlagen der folgenden Abbildungen sind direkte Pausen von Registrierblättern.

Abb. 3 zeigt die Registrierung eines Versuches mit ungefiltertem Licht der Hg-Hochdrucklampe (Kurve 1 und 2). Nach Aufnahme der Kurven 1 und 2 ist die Lampe gelöscht worden (Kurve 3). Zum Vergleich ist als Kurve 4 die thermische Ionen-Emission mit dem Gleichstromverstärker aufgezeichnet worden.

Für die Kurven 1, 2 und 3 ist der Ordinaten-Maßstab: $1 \mathrm{~cm}=5 \cdot 10^{-10}$ A, für Kurve $4: \quad 1 \mathrm{~cm}=3,75$ $\cdot 10^{-7} \mathrm{~A}, \mathrm{~d} . \mathrm{h}$. das Amplitudenverhältnis ist etwa $1: 1000$.

Einen ersten Überblick über die Abhängigkeit von Wellenlänge und Bedeckungsgrad gibt die Abb. 4. Hier zeigen die Kurven (die Numerierung erfolgte in der Reihenfolge der Aufzeichnung) 1, 2, 3 und 7 den Photoeffekt unter Verwendung eines Glasfilters (kurzwellige Grenze $3000 \AA$ ). Diese Kurven sind bei fester PlatinTemperatur und steigenden Ofentemperaturen gemacht, d. h. in der Reihenfolge der Numerierung steigt die

\footnotetext{
7 Mehrmals kurzzeitig bis $700{ }^{\circ} \mathrm{C}$.
} 
Abb. 3. Photoeffekt der Ionenemission im Licht der ungefilterten Hg-HochdruckDampflampe. Platin-Temperatur: $500{ }^{\circ} \mathrm{C}$; Ofen-Temperatur: Kurve 1: $160^{\circ} \mathrm{C}$, Kurve 2: $154{ }^{\circ} \mathrm{C}$; Kurve 1 und 2: Lichtsynchron gleichgerichteter $17 \mathrm{~Hz}$-Wechselstrom; Kurve 3: Wie 1 und 2, jedoch bei gelöschter Lampe; Kurve 4: Ionenstrom, gemessen mit Gleichstromverstärker.

Abb. 4. Photoeffekt der Ionenemission, Versuche mit Interferenz-Filtern. Platin-Temperatur: $480^{\circ} \mathrm{C}$, Ofen-Temperatur bei Kurve 1: $133^{\circ} \mathrm{C}$ (Glas) ; Kurve 2: $140{ }^{\circ} \mathrm{C}$ (Glas) ; Kurve 3 : $152{ }^{\circ} \mathrm{C}$ (Glas); Kurve 4: $164{ }^{\circ} \mathrm{C}(5430 \AA)$; Kurve $5: 166{ }^{\circ} \mathrm{C}(5880$ $\AA ̊ \AA)$; Kurve $6: 172{ }^{\circ} \mathrm{C}(6160 \AA ̊)$; Kurve 7: $173{ }^{\circ} \mathrm{C}$ (Glas) ; Kurve 8: $173^{\circ} \mathrm{C}(7750 \AA$ ) $)$. Das Glasfilter hatte eine kurzwellige Durchlaßgrenze von $3000 \AA \AA$, die anderen Wellenlängen-Angaben beziehen sich auf die Mitte des Durchlaßbereiches der Interferenzbandfilter. Die Durchlässigkeit der Interferenzfilter betrug im Maximum rund $50 \%$, die Halbwertsbreite rund $200 \AA \AA$ A. - Kurven 4, $5,6,8$ liegen in dem gestrichelten Band, ohne sich vom Untergrund zu unterscheiden.
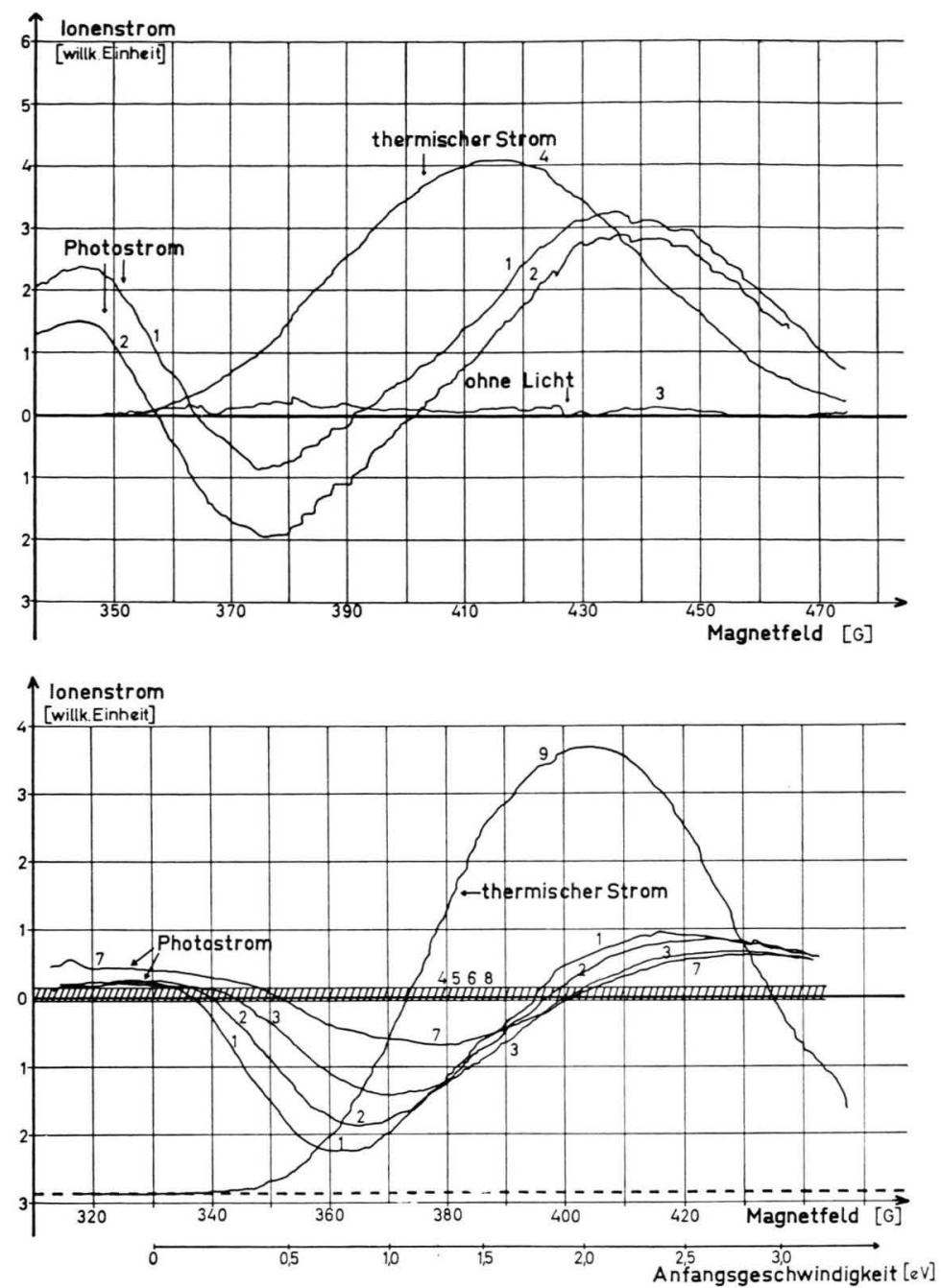

Anzahl der sekundlich auf die Oberfläche auftreffenden Kalium-Atome.

Die Kurven 4, 5, 6 und 8 dieser Abb. sind mit verschiedenen, in der $\mathrm{Abb}$. angegebenen Interferenzfiltern aufgenommen worden. Durch die Schraffierung soll angedeutet werden, daß in den so erfaßten Wellenlängenbereichen kein erkennbarer Einfluß des Lichtes auf den Ionenstrom vorlag. Zum Vergleich zeigt Kurve 9 wieder den mit dem Gleichstromverstärker registrierten thermischen Ionenstrom.

Man erkennt durch Vergleich der Kurven 1, 2, 3 in Abb. 3 und der Kurven 1, 2, 3, 7 der Abb. 4 mit den durch „ohne Licht“ gekennzeichneten Kurven, daß ein vom Licht ausgelöster Strom auftritt. Dieser Strom ist dem Licht synchron (gesteuerter Gleichrichter). In beiden Abbildungen ist das Vorzeichen des Stromes rechts vom Maximum der thermischen Emission positiv. In diesem Gebiet wird also die Ionenemission aus der Kugel durch das Licht vergrößert. Links vom thermischen Maximum ist in einem gewissen Gebiet das Vor- zeichen des Stromes negativ, hier wird dementsprechend die Ionenemission der Kugel durch das eingestrahlte Licht verkleinert.

Darüber hinaus ist in Abb. 3, also bei ungefiltertem Licht, ganz links nochmals eine Vergrößerung der Emission durch Licht festzustellen. In diesem Gebiet liegt keine thermische Emission vor. Die Lage des Maximums in diesem Gebiet entspricht einer Energie der Ionen beim Verlassen der Oberfläche von etwa 0 Volt.

\section{Fehlerquellen}

Eine Reihe von denkbaren Fehlerquellen, die eine Photoemission von Ionen vortäuschen könnten, wurde durch folgende Versuche ausgeschlossen:

\section{Streulicht}

Streulicht könnte in der ersten Stufe des Multipliers Elektronen auslösen und damit einen lichtsynchronen 
Strom vortäuschen. Diese Gefahr besteht besonders, weil nicht ausgeschlossen werden kann, daß die Bleche des Multipliers sich im Laufe der Versuche mit einer Monoschicht Kalium (Dampfdruck bei Zimmertemperatur $2 \cdot 10^{-8} \mathrm{~mm} \mathrm{Hg}$ ) bedecken.

Die Wirksamkeit der zur Abschirmung des Streulichtes vorgesehenen Blendenanordnung und des „schwarzen Körpers" kann man auf zweierlei Weise prüfen. Einmal kann man die Platinkugel auf negatives Potential legen, so daß bei sonst gleichen Verhältnissen keine Ionen aus der Kugel gelangen können. Da hierbei am Multiplier kein $17 \mathrm{~Hz}$-Strom auftritt, emittiert die erste Dynode keine Photoelektronen.

Darüber hinaus zeigt auch die Tatsache, daß bei kleinen und großen Magnetfeldern der Strom auf Null absinkt, daß kein Streulichteinfluß vorliegt.

\section{Photoionisation im K-Dampf}

Eine Photoionisation des in der Pt-Kugel vorhandenen K-Dampfes kann ausgeschlossen werden, da die vom Glasfilter durchgelassene Strahlung eine kurzwellige Grenze von $3000 \AA$ hat, während zur Photoionisation entsprechend der Ionisierungsspannung von $4,380 \mathrm{~V}$ $2840 \AA$ erforderlich wären.

\section{Elektronenstoß-Ionisation}

Es ist denkbar, daß eventuell außerhalb der Kugel niedergeschlagenes Kalium eine Photokathode bildet. Streulicht könnte hier Elektronen auslösen, welche in die auf positiver Spannung liegende Pt-Kugel gelangen und dort Stoß-Ionisation bewirken könnten.

Jedoch ist diese Störung für alle Herkunftsorte von Elektronen außerhalb der Kugel wegen der Ablenkung durch das Magnetfeld unwahrscheinlich.

Sicher auszuschließen ist der Effekt jedoch durch Variation der Ziehspannung. Diese Spannung wurde zwischen 2 und $15 \mathrm{~V}$ (Ionisierungsspannung des K $4,4 \mathrm{eV}$ ) variiert. Dabei konnte keine Abhängigkeit der Photo-Effekte von der Ziehspannung beobachtet werden, die auf Einsetzen einer Stoß-Ionisation schließen ließe.

\section{Diskussion}

Aus den beschriebenen Versuchen ergibt sich folgender Sachverhalt:

1. In einer Adsorptionsschicht von Kalium auf Platin tritt ein photoelektrischer Effekt auf, der in der Auslösung positiver Kalium-Ionen durch Einstrahlung von Licht besteht.

2. Die Geschwindigkeit der durch den Photoeffekt emittierten Ionen ist gegenüber der Geschwindigkeit thermisch emittierter Ionen verschoben.

3. Die Emission positiver Kalium-Ionen erfolgt bei größeren Wellenlängen als die Ionisation von Kalium im Dampf.
4. Die Größe des photoelektrischen Stromes und die Geschwindigkeitsverteilung der emittierten Ionen hängen von der Temperatur des Platins und der Anzahl der pro Sekunde auf die Platin-Oberfläche auftreffenden Kalium-Atome ab.

5. Neben der lichtsynchronen Emission von Ionen tritt an anderer Stelle der Geschwindigkeitsverteilung eine ebenfalls lichtsynchrone Verringerung des thermischen Ionenstromes auf. Für diese Verringerung gelten sinngemäß ebenfalls die Feststellungen unter Punkt 3 und 4.

Aus diesen Befunden ergibt sich eindeutig, daß die beobachteten Photoeffekte auf einem Oberflächenprozeß beruhen. Zum vollen Verständnis des Effektes sind ausführliche Messungen der Abhängigkeit von der Temperatur und vom Bedeckungsgrad der Oberfläche mit Kalium erforderlich. Diese Messungen sollten mit wenigstens annähernd monochromatischem Licht vom nahen Ultrarot bis ins Ultraviolett durchgeführt werden. Allerdings liegt die Schwierigkeit in der Erzeugung monochromatischen Lichtes ausreichender Intensität, das über den ganzen Wellenlängenbereich kontinuierlich variiert werden kann.

Weiterhin wäre es wünschenswert, Einkristallflächen verschiedener Metalle zu verwenden und den Einfluß von Fremdgasen zu untersuchen. Zur Frage der Reinheit der Platin-Oberfläche sei bemerkt, daß es sich bei den bisher ausgeführten Versuchen sicherlich nicht um eine „ideal reine“ Oberfläche im Sinne der Ultra-Hochvakuum-Technik gehandelt hat. Andererseits kann aber angenommen werden, da $\beta$ bei den angewendeten Ausheiztemperaturen und durch tagelange Einwirkung von Kaliumdampf auf das $500{ }^{\circ} \mathrm{C}$ heiße Platin eine Oberfläche entsteht, deren Adsorptionsverhalten demjenigen einer reinen Oberfläche mindestens ähnlich ist.

Betrachtet man die bisher gemachten Beobachtungen, so kommt man zu der Auffassung, daß von den eingangs angeführten drei Möglichkeiten eines Lichteinflusses die elektronische Anregung des adsorbierten Komplexes die wahrscheinlichste ist. Mit dieser Arbeitshypothese ergibt sich folgende, an Hand von Abb. 5 erläuterte Erklärung für das Zustandekommen der beobachteten Effekte.

Vereinfachend werden die Verhältnisse an nur einer der Flächen betrachtet, aus denen sich die polykristalline Oberfläche des Platins zusammensetzt. Außer der Ortskoordinate $(x)$ ist als Abszisse noch die Häufigkeit $(\Phi)$ aufgetragen, mit der Zustände 
bestimmter Energie $(E)$ besetzt sind. In Abb. 5 a ist schematisch die Energieverteilung des adsorbierten Kaliums eingetragen. Die tatsächliche Form der Verteilung ist vermutlich stark unsymmetrisch.

Daneben ist (Abb. 5 b) die Energieverteilung der von der Oberfläche emittierten Ionen in unendlicher Entfernung von der Oberfläche aufgetragen. Diese Energieverteilung hängt mit der Verteilung an der Oberfläche nahe zusammen.

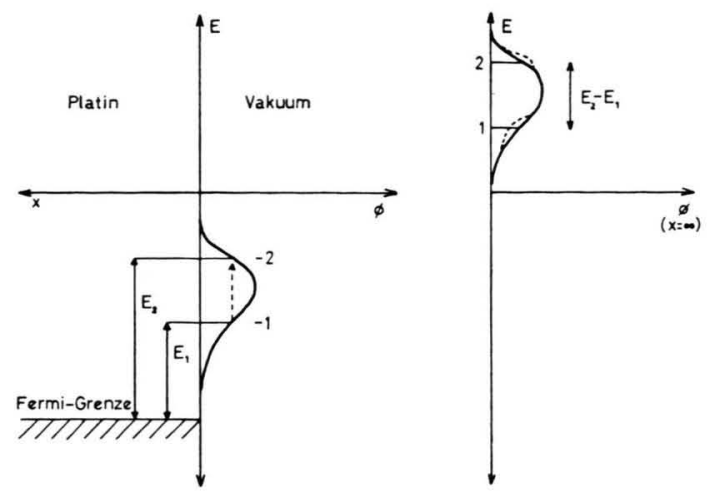

Abb. 5. Schematische Darstellung der Energieverteilung: a) adsorbiertes Kalium an der Pt-Oberfläche, b) emittierte Ionen in unendlicher Entfernung von der Oberfläche.

Beim Ionisationsvorgang an der Oberfläche wird die Ionisierungsarbeit geleistet und die Elektronenaustrittsarbeit gewonnen. Beide Arbeiten sind, unter anderem wegen des Einflusses adsorbierter Nachbarn, stark variierende Funktionen des Ionisierungsortes $^{8}$. Die im Ionisationsprozeß gewonnene Energie steht zur Entfernung des Ions ins Unendliche zur Verfügung. Hierbei müssen die Bildkraft und die anderen Adsorptionskräfte überwunden werden. $\mathrm{Da}$ in unserem Falle die Bildkraft die anderen Kräfte bei weitem überwiegt ${ }^{3}$, besteht die Energie der Ionen im Unendlichen im wesentlichen aus der an der Oberfläche gewonnenen Energie, vermindert um die Bildenergie.

In Abb. 5 a ist für zwei Stellen (1 und 2) der Verteilung an der Oberfläche die bei der Ionisation jeweils gewinnbare Energie durch die Pfeile $E_{1}$ und $E_{2}$ gekennzeichnet. Nach Emission von Ionen mit einer $E_{1}$ bzw. $E_{2}$ entsprechenden Energie ist die Größe der Differenz $\left(E_{2}-E_{1}\right)$ im Unendlichen sehr nahe gleich der Differenz $\left(E_{2}-E_{1}\right)$ an der Ober-

8 G. N. Shuppe, P. Sytaya u. M. Kadyrow, Izv. Akad. Nauk, SSSR, Ser. Fiz. 20, 1142 [1956]. fläche, weil die Bildkraft für beide Fälle annähernd gleich groß sein dürfte.

Tritt nun durch optische Anregung ein adsorbiertes Atom ${ }^{10}$ vom Zustand 1 in den Zustand 2 über, so erscheint es nach der Emission als Ion bei höheren Energien und vergrößert den Strom der thermisch mit dieser Energie emittierten Ionen. Umgekehrt wird es an derjenigen Stelle der Energieverteilung der emittierten Ionen fehlen, wo thermisch die Ionen aus dem Zustand 1 emittiert werden. Die auf diese Weise veränderte Form der Häufigkeitsverteilung ist in Abb. $5 \mathrm{~b}$ gestrichelt eingezeichnet. Damit wäre erklärt, wieso es dazu kommt, daß bei kleinen Energien eine Schwächung, bei größeren dagegen eine Verstärkung des Ionenstroms durch das eingestrahlte Licht eintritt. (Vgl. Abb. 3 und 4.)

Aus dem Energieunterschied der Ionen zwischen dem Maximum der Stromvergrößerung und dem Maximum der Stromverkleinerung kann man auf die Differenz $\left(E_{2}-E_{1}\right)$ an der Oberfläche schließen. Solange keine Messungen der Wellenabhängigkeit vorliegen, kann für diesen Energieunterschied, der durch Absorption von Licht in der Adsorptionsschicht hervorgerufen wird, nur eine Abschätzung angegeben werden. In Abb. 4 ist unter der Magnetfeld-Skala noch eine zweite Einteilung in $\mathrm{eV}$ angegeben. Diese Energien sind aus Masse, Bahnradius und der angelegten Beschleunigungsspannung berechnet. Bei der geringen Auflösung des Instrumentes ist die Lage des Nullpunktes dieser Skala um $\pm 0,2 \mathrm{eV}$ unsicher. Dieser Fehler wirkt sich bei höheren Anfangsenergien wegen der Wurzelabhängigkeit stärker aus. Immerhin kann der Betrag $E_{2}-E_{1}$ zu etwa 1,5 eV abgeschätzt werden.

Nach diesen halb-quantitativen Betrachtungen scheinen die beiden anderen in der Einleitung erwähnten Möglichkeiten für das Zustandekommen des Lichteffektes unwahrscheinlich zu sein. Die Anregung von Schwingungsenergie der adsorbierten Atome sollte nur Energiedifferenzen in der Größenordnung 0,1 eV liefern. Eine vom Licht ausgelöste Änderung in der Besetzung der elektronischen Oberflächenterme des Platins erscheint ebenfalls unwahrscheinlich, weil damit die nebeneinander auftretende Vergrößerung und Verringerung des thermischen Ionenstromes schwer erklärbar wäre. Jedoch kann

10 bzw. ein Adsorptionskomplex. 
ein solcher Effekt nach den bisher vorliegenden Messungen nicht völlig ausgeschlossen werden.

Wenn sich die hier dargelegte Auffassung weiter bestätigen läßt, wird es möglich sein, die bei der Adsorption eintretende Änderung des Termsystems von Atomen näher zu untersuchen.
Ich danke Herrn Prof. Dr. R. Haul für Interesse und Förderung dieser Arbeit sowie für ausführliche und wertvolle Diskussionen. - Der Deutschen Forschungsgemeinschaft habe ich für die Bewilligung der Geräte und dem Fonds der Chemie für großzügige Förderung verbindlich zu danken.

\title{
Die Energieverteilüng schneller Photoneutronen aus Blei*
}

\author{
Von Hans Breuer \\ Aus dem Max-Planck-Institut für Biophysik, Frankfurt a. M. \\ (Z. Naturforschg. 17 a, 584 -590 [1962] ; eingegangen am 5. April 1962)
}

\begin{abstract}
Es wurde das Spektrum der unter $90^{\circ}$ emittierten Photoneutronen aus Blei mit Hilfe eines LiJ-Spektrometers gemessen. Das Spektrum setzt sich aus zwei Anteilen zusammen: Der größte Teil (rund 89\% aller emittierten Neutronen) stammt aus der Emission, die mit der Verdampfungstheorie von $W_{\text {EISSKOPF }}$ beschrieben werden kann und zeigt eine MAxweLL-Verteilung mit einem Maximum bei $0,9 \mathrm{MeV}$. $11 \%$ aller Neutronen können mit dem sog. „direkten“ Kernphotoeffekt erklärt werden und folgen einer Energieverteilung mit einem Maximum zwischen 4 und $5 \mathrm{MeV}$.
\end{abstract}

In den letzten Jahren hat das Photoneutronenspektrum von schweren Kernen zunehmend an Interesse gewonnen. Da es sich hierbei um Neutronen im MeV-Bereich handelt, wurden in erster Linie Photoplatten als Spektrometer benutzt ${ }^{1,2}$. Das hervorragende Energieauflösungsvermögen der Photoplatte ist allerdings mit einer recht mühsamen Auswertung der Spuren der Rückstoßprotonen verbunden. In der vorliegenden Arbeit wurde versucht, diese mühsame Auswertung zu umgehen; es muß dabei allerdings eine Verschlechterung des Energieauflösungsvermögens in Kauf genommen werden.

Abb. 1 zeigt das Ansprechvermögen verschiedener Meßmethoden als Funktion der Neutronenenergie. Die Darstellung kann keinen Anspruch auf Vollständigkeit erheben, enthält aber die wesentlichen, auf die geringen Intensitäten eines Betatrons abgestimmten Möglichkeiten der Spektrometrie schneller Neutronen. Wie aus Abb. 1 hervorgeht, ist ein Spektrometer auf der Basis der $\mathrm{Li}^{6}(\mathrm{n}, \alpha)$ T-Reaktion optimal, wenn man von der Photoplatte absehen will.

\section{Das Spektrometer}

Die $\mathrm{Li}^{6}(\mathrm{n}, \alpha) \mathrm{T}$-Reaktion ist an verschiedenen Stellen ${ }^{3-6}$ beschrieben worden.

Der hier benutzte Spektrometerkristall ist $2 \mathrm{~mm}$ dick und hat einen Durchmesser von $1 \times \frac{1}{2}$ inch. Er ist mit

\footnotetext{
* Gewidmet Herrn Prof. Dr. Dr. h. c. Dr. h. c. B. Rajewsky zum 25jährigen Bestehen des „Max-Planck-Instituts für Biophysik".

1 M. E. Toms u. W. E. Stephens, Phys. Rev. 108, 77 [1957].

2 R. F. Askew u. A. P. Batson, Nucl. Phys. 20, 408 [1960].
}

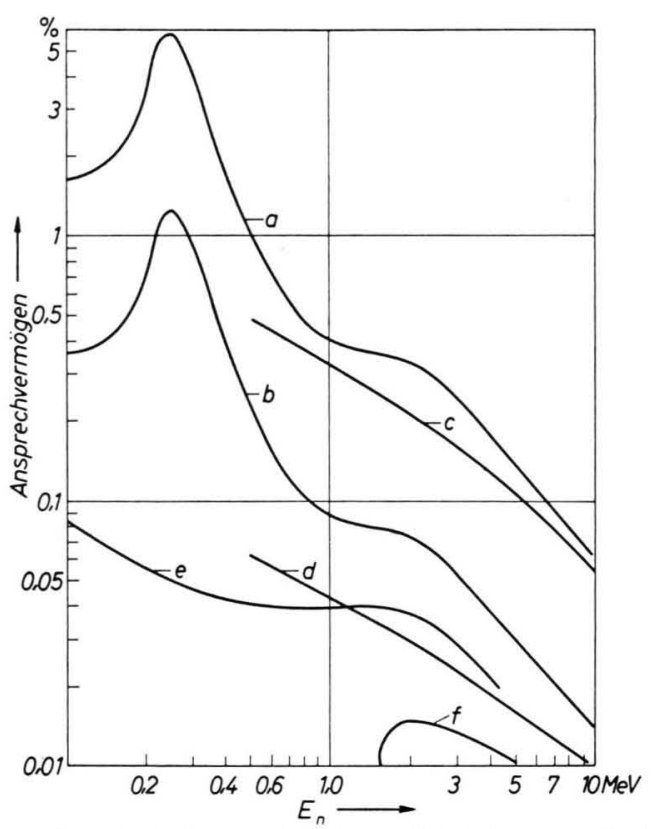

Abb. 1. Vergleich der verschiedenen Methoden zur Spektrometrie schneller Neutronen. $\left(a=\mathrm{Li}^{6} \mathrm{~J}\right.$-Kristall $10 \mathrm{~mm}$ dick ${ }^{6}$, $\mathrm{b}=\mathrm{Li}^{6} \mathrm{~J}$-Kristall $2 \mathrm{~mm}$ dick, $\mathrm{c}=200 \mu$-Photoplatte, $\mathrm{d}=$ Protonenzähler mit Kollimator, $\mathrm{e}=\mathrm{He}^{3}$ (n,p) -Zähler, $\mathrm{f}=$ schnelle Koinzidenzen; c, d, e, f nach C. D. Swartz, NYO-3863, 1954.

einem $8 \mathrm{~cm}$ langen Quarzlichtleiter verbunden (Hersteller: $\mathrm{H}$ a r s h a w Co. Cleveland, Ohio). Es wurde ein nur $2 \mathrm{~mm}$ dicker Kristall gewählt, um die durch

${ }^{3}$ G. R. Keepin u. J. H. Roberts, Rev. Sci. Instrum. 21, 163 [1950].

${ }^{4}$ R. B. Murray u. J. Schenck, Bull. Amer. Phys. Soc. 2, 296 [1956].

${ }^{5}$ R. B. Murray, Report CF 56-11-5, ORNL, 1956.

${ }^{6}$ R. B. Murray, Nucl. Instrum. 2, 237 [1958]. 